\title{
The Impact of Duration of Complaints on Successful Outcome of Sacral Neuromodulation
}

\author{
Ranjana Jairam ${ }^{a, b}$ Jamie Drossaerts ${ }^{a, b} \quad$ Gommert van Koeveringe ${ }^{a-c}$ \\ Philip van Kerrebroeck ${ }^{\mathrm{a}-\mathrm{c}}$ \\ a Department of Urology, Maastricht University Medical Centre (MUMC+), bSchool of Mental Health and \\ Neuroscience (MHeNS), Maastricht University, and 'Pelvic Care Centre Maastricht, Maastricht University \\ Medical Centre, Maastricht, The Netherlands
}

\section{Keywords}

Non-obstructive urinary retention - Overactive bladder .

Sacral neuromodulation · Symptom duration - Underactive bladder

\begin{abstract}
Objectives: The study aimed to evaluate whether the duration of complaints in patients with overactive bladder syndrome or non-obstructive urinary retention predicts the outcome of sacral neuromodulation (SNM). Methods: All patients that underwent a SNM test period evaluation between 2011 and 2014, were included in this study. The duration of complaints was listed in 3 categories: (a) 0-5 years, (b) 5-10 years and (c) 10 years or longer. Analyses with chi square tests were performed to evaluate whether the duration of complaints are associated with outcome of SNM. Results: In total, 130 patients were included. Most patients had a complaint duration of $0-5$ years $(n=60)$. The test period was successful in $56 \%(n=74)$ of the total group. Analyses showed that the duration of complaints is not significantly associated with outcome of SNM ( $p=0.752)$, even when subdivided per indication, and also when possible confounders such as age at test and indication are taken into account $(p=0.720)$. Conclusion: Based on the results of this study, there is no relationship between duration of complaints and SNM out-
\end{abstract} www.karger.com/uin

\section{KARGER}

E-Mail karger@karger.com
(C) 2017 The Author(s)

Published by S. Karger AG, Basel

This article is licensed under the Creative Commons AttributionNonCommercial-NoDerivatives 4.0 International License (CC BYNC-ND) (http://www.karger.com/Services/OpenAccessLicense). Usage and distribution for commercial purposes as well as any distribution of modified material requires written permission. come. SNM seems to remain a feasible treatment option, despite of possible anatomical or physiological changes within the lower urinary tract.

(c) 2017 The Author(s)

Published by S. Karger AG, Basel

\section{Introduction}

Sacral neuromodulation (SNM) is a treatment option in patients with overactive bladder syndrome $(\mathrm{OAB})$ with or without urinary urgency incontinence and non-obstructive urinary retention (NOR) if conservative treatment fails.

In order to predict which patients will respond to treatment with SNM and who will achieve an optimal SNM test outcome, selection of patients is crucial. Success rates vary between 60 and $80 \%$ [1]. It still remains unclear which exact patients will benefit from SNM treatment, and thus a test period is necessary. However, several studies identified predictive factors, but for most patients only a successful test period predicts positive treatment outcome [2, 3]. Recently, it was addressed that ambulatory urodynamic studies can be of guidance in adequate pa-

J.D. and R.J. contributed equally to this work.

Ranjana Jairam

Department of Urology, Maastricht University Medical Centre (MUMC+)

P. Debyelaan 25, POB 5800

NL-6202 AZ Maastricht (The Netherlands)

E-Mail r.j.jairam@outlook.com 
tient selection, as the detection of a genuine acontractile bladder can predict SNM failure [4].

Most patients who are eligible for SNM have had complaints for many years, refractory to conservative treatment such as bladder training, pelvic floor exercise, behavioral therapy, and antimuscarinics or a beta-3-adrenergic receptor agonist before SNM is offered. Some patients are refractory even to secondary therapies like botulinum toxin bladder injections or percutaneous tibial nerve stimulation.

We questioned whether the duration of complaints before performing SNM could predict treatment failure.

Considering the time frame of duration of specific voiding or storage symptoms, and the subsequent chances of success of SNM, very little is known. Only one study investigated the duration of complaints as a primary outcome measure [5]. Most studies reported on duration of complaints before SNM treatment only as a secondary outcome measure, and were investigating other possible predictive factors in SNM outcome [3, 6-10]. These studies mostly consist of small groups of patients with either NOR or OAB and with a variable range of duration of complaints (up to 5 years). The results of these studies are contradictory which makes it useful to investigate a large group of patients with a longer duration of complaints. Also, in order to identify which patients will have benefit of treatment with SNM, it is important to understand the underlying pathology.

\section{Pathophysiology of the Overactive Bladder}

Detrusor overactivity (DO) is a urodynamic observation, which often coincides with $\mathrm{OAB}$, and is characterized by involuntary detrusor contractions during the filling phase.

There are 3 hypotheses on the emergence of DO. The neurogenic hypothesis states that DO arises from generalized, nerve-mediated excitation of the detrusor muscle which can occur after brain damage $[11,12]$. Furthermore, the myogenic hypothesis states that coordinated myogenic contractions of the detrusor occur due to partial denervation of the detrusor [13].

The urothelium also seems to be contributing to the pathogenesis of $\mathrm{OAB}$ - changes in urothelial receptor function and neurotransmitter release may lead to the enhancement of involuntary bladder contractions (urothelium-based hypothesis) [14].

\section{Pathophysiology of NOR}

Chronic urinary retention without evident obstruction poses a great challenge for management, since the pathophysiology is poorly understood. There are differ- ent causes of NOR. It can be caused by detrusor underactivity: this can be neurogenic (when there is a failure of initiating and maintaining a normal detrusor contraction) or myogenic (when the bladder smooth muscle function is impaired due to, e.g., overdistension, infection, or fibrosis). Other functional etiologies playing a role in NOR are the following: detrusor bladder neck dyssynergia, detrusor external sphincter dyssynergia, dysfunctional voiding, and non-relaxing urethral sphincter obstruction [11].

It is proposed that in an overactive bladder, anatomical and/or physiological changes can occur. Since signaling in the urothelium seems to play a role in the pathogenesis of overactive bladder, this might be of importance in (irreversible) alterations of the bladder wall. Also, studies show alterations in smooth muscle, which occur in an overactive bladder, may be a consequence of denervation of the detrusor which can contribute to DO $[15,16]$. Animal research shows that SNM prevents alterations of the bladder wall [17]. This indicates that SNM may be able to compensate for bladder wall changes. However, the exact mechanism of action is still not well understood. Different working mechanisms are proposed. One of the theories is that SNM might exert its effect in the guarding reflexes by altering the bladder afferent signaling which leads to restoration of voluntary micturition [18]. It is also believed that SNM inhibits the ascending limb of the micturition reflex. As a result, the signaling input from the bladder to the pontine micturition center is blocked and involuntary micturition is prevented in patients who suffer from OAB [19].

In addition, SNM also seems to inhibit the excitatory outflow to the urethral outlet and, therefore, promotes bladder emptying [18]. However, it is observed that SNM can also have its effect by an increase in detrusor contractility that is sufficient to overcome an overactive urethral sphincter [20].

Furthermore, it is observed that early SNM in patients with complete spinal cord injury could prevent the development of DO and urinary incontinence since it may preserve nerve plasticity, such that $\mathrm{C}$-fibers remain silent and DO is avoided [21-23]. This observation may indicate modulation at the level of the spinal cord itself as well as the importance of early treatment with SNM.

Based on the pathophysiology of OAB and NOR and the structural changes that occur in the bladder wall, we hypothesize that a longer duration of complaints can result in irreversible structural and/or cellular changes which may lead to less successful treatment outcome[24, $25]$. The aim of this study is to determine whether there
52

Urol Int 2017;99:51-55

DOI: $10.1159 / 000456079$
Jairam/Drossaerts/van Koeveringe/ van Kerrebroeck 
is a predictive value of the duration of complaints on SNM test outcome. Furthermore, we investigated if the duration of complaints can predict the long-term success.

\section{Methods}

The study population consisted of patients who underwent a SNM test period evaluation between 2011 and 2014 at the Maastricht University Medical Centre. During history taking at initial out-patient clinic visit, all patients were asked for the duration of their complaints. This data were collected in a database and used for this retrospective study.

A total of 130 patients with refractory urgency and frequency, with or without urgency incontinence or urinary retention who underwent a test period evaluation, were included. The test period lasted 4 weeks. The results of the test period were evaluated using data from voiding diaries. Success was considered as a symptom improvement of $50 \%$ or more. Duration of complaints in months were extracted from patient files and were defined as the time between the start of complaints till the date of the SNM test period. We listed the duration in 3 categories: (a) 0-5 years, (b) 5-10 years and (c) 10 years or longer. This cutoff was chosen arbitrarily since there is no definition of a short or long duration of complaints.

\section{Statistical Analysis}

For statistical analysis SPSS, IBM corporation, version 22, was used. Descriptive statistics were performed and presented as mean \pm SD. Analyses with chi square tests were done to evaluate whether the duration of complaints before SNM was associated with success of SNM during test period. Fisher's exact test was used to evaluate if the duration of complaints was associated with outcome 1 year after implantation of the IPG. Results were considered statistically significant when $p<0.05$. Logistic regression analysis was used in order to evaluate if the duration of complaints is associated with outcome of SNM, when age and SNM indication were also taken into account.

\section{Results}

A total of 130 patients were included - 42 male and 88 female patients. Mean age at the moment of SNM test was 48.9 (SD 14.5). Other patient characteristics are shown in Table 1. Mean duration of complaints was 8.08 years (SD 8.54). Of all the patients enrolled, $51 \%$, had OAB-wet, 12 (9\%) had OAB-dry, and 52 (40\%) were NOR patients. Since the OAB-dry group was small, OAB-wet and -dry were addressed as one group (OAB). SNM test period was successful in $56 \%(n=74)$ of the total group. In the OAB patients, $68 \%$ was successfully treated and in the NOR group $40 \%$. The mean duration of complaints in months in the $\mathrm{OAB}$ and NOR patients was 115 and 73.5 months, respectively. Most patients had a complaint duration of $0-5$ years $(n=$ 61),28 patients had a complaint duration of 5-10years, and 41 patients had complaints since at least 10 years (Fig. 1).

Are Symptom Duration and Rate of Success Related?

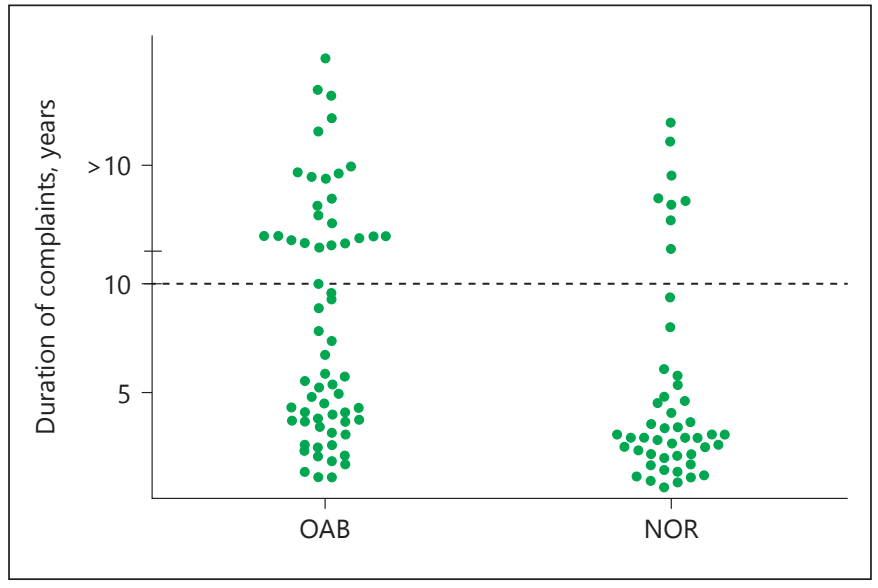

Fig. 1. Duration of complaints (in years) in all patients. Data were plotted in months.

Table 1. Characteristics of patients undergoing SNM test period between 2011 and 2014

\begin{tabular}{ll}
\hline Patient characteristics & Number of patients (\%) \\
\hline Sex & \\
$\quad$ Male & $42(32)$ \\
Female & $88(68)$ \\
Indication & \\
OAB & $78(60)$ \\
NOR & $52(40)$ \\
History of & \\
Stress incontinence surgery & $26(20)$ \\
Hysterectomy & $27(21)$ \\
Other abdominal surgery & $34(26)$ \\
Neurological disease & $8(6)$ \\
IDP surgery & $9(7)$ \\
Psychiatric disease & $22(17)$ \\
\hline
\end{tabular}

Table 2. Association between duration of complaints and success of test-stimulation

\begin{tabular}{llll}
\hline $\begin{array}{l}\text { Duration of complaints, } \\
\text { years }\end{array}$ & $n$ & $\begin{array}{l}\text { Test success, } \\
(\%)\end{array}$ & $p$ value \\
\hline $0-5$ & 61 & $33(54)$ & 0.789 \\
$5-10$ & 28 & $16(57)$ & \\
$>10$ & 41 & $25(61)$ & \\
\hline
\end{tabular}

In the $\mathrm{OAB}$ group, there were significantly more patients who had complaints for more than 10 years $(p=0.004)$.

Analyses with chi square tests showed that the duration of complaints is not significantly associated with the success of SNM ( $p=0.789$; Table 2$)$. When subdivided per indication also, no significant associations were present $(p=0.340$ and $p=0.525$; Table 3$)$. Logistic regression 
Table 3. Association between duration of complaints and success of test-stimulation in OAB and NOR patients

\begin{tabular}{|c|c|c|c|c|c|c|}
\hline \multirow[t]{2}{*}{ Duration of complaints, years } & \multicolumn{3}{|c|}{ OAB patients } & \multicolumn{3}{|c|}{ NOR patients } \\
\hline & $n$ & test success, $n(\%)$ & $p$ value & $n$ & test success, $n(\%)$ & $p$ value \\
\hline $0-5$ & 27 & $21(78)$ & & 34 & $12(35)$ & \\
\hline $5-10$ & 19 & $11(58)$ & 0.340 & 9 & $5(56)$ & 0.525 \\
\hline$>10$ & 32 & $21(66)$ & & 9 & $4(44)$ & \\
\hline
\end{tabular}

analysis showed that duration of complaints is not significantly related to success of SNM, even when age at test and SNM indication were also taken into account $(p=$ 0.720 ). Furthermore, the duration of complaints was not significantly associated with success 1 year after implantation $(p=0.203)$. Also, when subdivided per indication (OAB and NOR), no significant associations were found ( $p=0.456$ and $p=0.559$, respectively).

\section{Discussion}

Recent studies show that there are predictive factors that may influence the outcome in patients treated with SNM [2-4]. However, it is not known whether the duration of complaints predicts success in SNM. This is the first large study to investigate whether the duration of complaints predicts success in SNM. More importantly, in this study, there are many patients with an evident long duration of complaints (10 years or longer).

In 2002, a study screening for possible predictive factors by Scheepens et al. [3] found that a duration of complaints for more than 7 months was a predictor in SNM test outcome. However, they did not describe how they choose this cutoff. An abstract of Ghazwani et al. [7] which was presented at the International Continence Society (ICS) in 2012 found that patients with a successful SNM test outcome had a significantly shorter duration of complaints $(2.5 \pm 2$ vs. $6.2 \pm 3.8$ years, $p=0.003)$. They also concluded that the duration of complaints had a negative correlation in SNM test outcome $(r=-0.5)$. Sherman et al. [10] investigated SNM for urgency incontinence in women who had undergone stress incontinence surgery. They found that a duration less than 4 years between stress incontinence surgery and test stimulation seemed to be predictive for a positive response to neuromodulation. The authors attribute this to unidentified pathologic or neurologic deterioration that occur, and state that success is limited when time to intervention is prolonged.
Our study shows that the duration of complaints is not significantly associated with SNM outcome. We also found that the indication for SNM (OAB-wet or -dry or NOR) did not show any significant association in outcome, even when duration of complaints was corrected for age. These findings extend those of Janknegt et al. [8] where no significant difference was seen in success rate when patients with urgency incontinence $<5$ years were compared to patients with urgency incontinence $>5$ years. Amundsen et al. [6] stated that there was no difference in duration of symptoms between the responders $(7 \pm$ 5 years) and non-responders ( $4 \pm 3$ years). More recently, Cerruto et al. [5] presented an abstract at the annual ICS meeting of 2012. They choose a cutoff point between short and long duration of symptoms of 4 years, and found that duration of symptoms was not significant in neither a decrease of micturition frequency nor incontinence episodes ( $p=0.621$ and $p=0.803$, respectively). Also, Saber-Khalaf et al. [9] retrospectively analyzed possible factors that might lead to a positive SNM test outcome. But although the duration of symptoms in the responders was less when compared to the failure group (3 vs. 7 years, respectively), this difference between groups was not significant $(p=0.368)$.

Up until now, some studies indicate that the duration of complaints is not significantly associated with SNM test outcome, while some studies do find a significant association. Most studies consisted of small groups of patients who had complaints for only a couple of years.

Our study is the first study consisting of a large number of patients, where a substantial group had complaints for more than 10 years. However, there are some limitations in our study. First, it is challenging to objectively determine the duration of symptoms in patients with $\mathrm{OAB}$ or NOR due to recall bias. Also, it is difficult to determine the exact duration of complaints since storage or voiding dysfunction might be present before patients manifest with the complaints.

Second, there is no definition of a short or long duration of complaints and the cutoff of the duration in the other
54

Urol Int 2017;99:51-55

DOI: $10.1159 / 000456079$
Jairam/Drossaerts/van Koeveringe/ van Kerrebroeck 
studies was chosen arbitrarily. We chose our cutoff values as it is probable that the bladder in patients with complaints for 5 years show more reversible structural changes than patients who have complaints for more than 10 years. However, there are no studies that confirm this statement.

Based on this large retrospective study, we may conclude that there is no significant association between the duration of complaints and SNM outcome. As mentioned before, for most patients, only a successful test period predicts positive treatment outcome. The results of this study indicate that even in patients with chronic symptoms it is useful to perform a SNM test period since the chance of a successful outcome is comparable to those who have a short duration of complaints. Since successful outcome in our study was not related to the duration of complaints, we suggest that if structural changes occur over time in the untreated underactive and overactive bladder, it might be possible that SNM facilitates restoration of these changes or allows to compensate[10,17]. Also, the SNM effect occurs in a relatively short time (days), and therefore, it is unlikely that the chronic changes in the bladder resolve within days. Since our study shows no difference in SNM outcome in patients with a short and long duration of complaints, this suggests that the duration of complaints might not be related to the success of SNM.

The use of SNM will enlighten more about the pathophysiology of OAB and NOR, which is often of idiopathic origin. In order to investigate if duration of complaints predicts SNM outcome, future work should also explore the influence of SNM on possible anatomical and physiological changes.

\section{Disclosure Statement} form.
All authors filled out an ICMJE conflicts of interest disclosure

\section{References}

1 van Kerrebroeck PE, van Voskuilen AC, Heesakkers JP, Lycklama a Nijholt AA, Siegel S, Jonas U, et al: Results of sacral neuromodulation therapy for urinary voiding dysfunction: outcomes of a prospective, worldwide clinical study. J Urol 2007;178: 2029-2034.

2 Koldewijn EL, Rosier PF, Meuleman EJ, Koster AM, Debruyne FM, van Kerrebroeck PE: Predictors of success with neuromodulation in lower urinary tract dysfunction: results of trial stimulation in 100 patients. J Urol 1994;152(6 pt 1):2071-2075.

3 Scheepens WA, Jongen MM, Nieman FH, de Bie RA, Weil EH, van Kerrebroeck PE: Predictive factors for sacral neuromodulation in chronic lower urinary tract dysfunction. Urology 2002;60:598-602.

4 Drossaerts J, Rademakers K, van Koeveringe G, Van Kerrebroeck P: The value of urodynamic tools to guide patient selection in sacral neuromodulation. World J Urol 2015;33: 1889-1895.

5 Cerruto MA, Curti P, D'Elia C, et al: Role of symptoms duration as prognosticator for sacral neuromodulation in refractory overactive bladder. ICS abstract, 2012.

6 Amundsen CL, Webster GD: Sacral neuromodulation in an older, urge-incontinent population. Am J Obstet Gynecol 2002;187: 1462-1465; discussion 1465.

7 Ghazwani Y, Elkelin M, Hassouna M: Sacral neuromodulation as a treatment option for post-hysterectomy urine retention; a long term follow-up. ICS/IUGA abstract, 2010.

8 Janknegt RA, Hassouna MM, Siegel SW, et al: Long-term effectiveness of sacral nerve stim- ulation for refractory urge incontinence. Eur Urol 2001;39:101-106.

9 Saber-Khalaf M, Abtahi B, Gonzales G, Helal M, Elneil S: Sacral neuromodulation outcomes in male patients with chronic urinary retention. Neuromodulation 2015;18:329334; discussion 334.

10 Sherman ND, Jamison MG, Webster GD, Amundsen CL: Sacral neuromodulation for the treatment of refractory urinary urge incontinence after stress incontinence surgery. Am J Obstet Gynecol 2005;193:2083-2087.

11 Wein A, Kavoussi L, Novick AC, Partin AW, Peters CA: Campbell-Walsh Urology, ed 10. Philadelphia, Elsevier, 2012, p 3757.

12 de Groat WC: A neurologic basis for the overactivebladder.Urology 1997;50(6A suppl):36-52; discussion 53-56.

13 Brading AF: A myogenic basis for the overactivebladder.Urology 1997;50(6A suppl):57-67; discussion 68-73.

14 Birder LA, de Groat WC: Mechanisms of disease: involvement of the urothelium in bladder dysfunction. Nat Clin Pract Urol 2007;4:46-54.

15 Elbadawi A, Yalla SV, Resnick NM: Structural basis of geriatric voiding dysfunction. III. Detrusor overactivity. J Urol 1993;150(5 pt 2): 1668-1680.

16 Turner WH, Brading AF: Smooth muscle of the bladder in the normal and the diseased state: pathophysiology, diagnosis and treatment. Pharmacol Ther 1997;75:77-110.

17 Comiter CV, Mazar C, Phull H, Salkini M: Chronic sacral nerve stimulation prevents detrusor structural and functional changes associated with bladder outlet obstruction-a rat model. Neurourol Urodyn 2010;29:783-788.
18 Leng WW, Chancellor MB: How sacral nerve stimulation neuromodulation works. Urol Clin North Am 2005;32:11-18.

19 de Groat WC, Theobald RJ: Reflex activation of sympathetic pathways to vesical smooth muscle and parasympathetic ganglia by electrical stimulation of vesical afferents. J Physiol 1976;259:223-237.

20 Kessler TM, La Framboise D, Trelle S, Fowler CJ, Kiss G, Pannek J, et al: Sacral neuromodulation for neurogenic lower urinary tract dysfunction: systematic review and meta-analysis. Eur Urol 2010;58:865-874.

21 Blok BF: Central pathways controlling micturition and urinary continence. Urology 2002; 59(5 suppl 1):13-17.

22 de Groat WC, Yoshimura N: Mechanisms underlying the recovery of lower urinary tract function following spinal cord injury. Prog Brain Res 2006;152:59-84.

23 Sievert KD, Amend B, Gakis G, Toomey P, Badke A, Kaps HP, et al: Early sacral neuromodulation prevents urinary incontinence after complete spinal cord injury. Ann Neurol 2010;67:74-84.

24 Zhou F, Li H, Zhou C, Lv H, Ma Y, Wang Y, et al: Structural and functional changes in gap junctional intercellular communication in a rat model of overactive bladder syndrome induced by partial bladder outlet obstruction. Exp Ther Med 2016;11:21392146.

25 Brierly RD, Hindley RG, McLarty E, Harding DM, Thomas PJ: A prospective controlled quantitative study of ultrastructural changes in the underactive detrusor. J Urol 2003;169: 1374-1378.
Are Symptom Duration and Rate of Success Related?
Urol Int 2017;99:51-55

DOI: $10.1159 / 000456079$ 